\title{
CDISC Other ADaM Dataset
}

National Cancer Institute

\section{Source}

National Cancer Institute. CDISC Other ADaM Dataset. NCI Thesaurus. Code C103375.

An ADaM analysis dataset that doesn't conform to the ADSL or BDS structure. 